\title{
An experiment in heat conduction using hollow cylinders
}

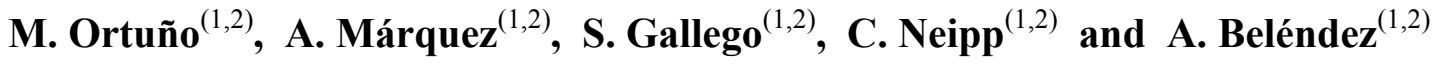 \\ (1) Departamento de Física, Ingeniería de Sistemas y Teoría de la Señal. \\ Universidad de Alicante. Apartado 99. E-03080 Alicante. SPAIN
}

(2) Instituto Universitario de Física Aplicada a las Ciencias y las Tecnologías.

Universidad de Alicante. Apartado 99. E-03080 Alicante. SPAIN

\begin{abstract}
An experimental apparatus was designed and built to allow students to carry out heat conduction experiments in hollow cylinders made of different materials as well as to determine the thermal conductivity of these materials. The evolution of the temperature difference between the inner and the outer walls of the cylinder as a function of time is analyzed and when the process reaches the steady state regime, the thermal conductivity can be easily calculated. Several materials such as wood, plastic and metals are considered and the values of their thermal conductivities experimentally obtained are compared with those given in the bibliography.
\end{abstract}

Keywords: Heat transfer; Heat conduction; Steady state regime; Thermal conductivity. 


\section{Introduction}

One of the topics studied in introductory physics and thermodynamics classes at university is heat transfer by conduction, which also has practical applications in thermal insulation problems usually studied in engineering and architectural degrees [1]. It is one of the three basic mechanisms of heat transfer and the most widely explained in undergraduate textbooks, with the statement of the basic Fourier law and the definition of parameters such as thermal conductivity or thermal resistance [2]. Conduction refers to the transfer of thermal energy between neighbouring molecules in a substance due to the existence of a temperature gradient. Heat conduction always takes place from a higher temperature region to a lower temperature region, and serves to equalize the temperature difference. Conduction takes place in solids, liquids and gases without any motion of matter. In solids, this mechanism of heat transfer is due to the combination of vibrations of the molecules in a lattice. In this way thermal energy is transmitted from the molecules of higher translational kinetic energy (higher temperature) to molecules of lower kinetic energy (lower temperature) without any mass transfer. In metals there is also a large number of free electrons which can move easily through the matter and these electrons contribute to the flow of heat by conduction. In gases and liquids, conduction is due to collisions and diffusion of the molecules during their random motion. Conduction is the most important heat transfer mechanism within a solid or between solid objects in thermal contact. This is due to the fact that the network of relatively fixed spatial relationships between atoms helps to transfer energy between them by vibration [3].

It is necessary to determine whether the heat transfer process is steady state or transient. When the heat flux in a system is independent of time, the temperature at any point in the system is also independent of time and then the regime is steady state. The process is said to be transient when the temperature at any point of the system is a function of time. In most university level physics textbooks there are many examples of heat conduction problems, mainly involving walls, rods, spheres and cylinders. Heat conduction in solids has been studied extensively for many years. Díaz-Aguilera [4] studied the heat conduction in one-dimensional solids, Ràfois and Ortín [5] presented an experimental realization of the problem of heat conduction along a one-dimensional metallic rod with Newtonian losses and Bee et al [6] analyzed realistic problems involving thermal conductivity. Transient heat conduction has been experimentally analyzed by Brody et al [7] using an optical set-up with 
a laser, while Laufer et al [8] presented a method to allow the temperature to be visualized in conducting solids using thermographic phosphors. Bacon et al [9] presented a range of experiments in heat conduction using liquid crystals suitable for upper level undergraduate laboratories and Brun and Pacheco [1] analyzed the reduction in heat transfer through a wall due to conduction, convection and radiation.

The aim of this paper is to present an experimental apparatus which allows heat conduction in hollow cylinders made of different materials to be studied. Furthermore, when the heat conduction process in these cylinders reaches the steady state, measurement of the temperature difference between the inner and outer wall of the cylinder allows us to determine the coefficient of thermal conductivity of the material of which the cylinder is made. These values are compared with those reported in the bibliography for the same materials. In summary, we present the results obtained from a laboratory experiment that students can carry out in an introductory physics course at university that includes teaching heat transfer.

\section{Theory}

\subsection{Heat conduction equation}

The thermal conductivity $(k)$ is used to quantify the ease with which a particular medium conducts. It is defined as the quantity of heat $(Q)$ transmitted in time $(t)$ through a thickness $(\Delta x)$, in a direction normal to a surface of area $(S)$, due to a temperature difference $(\Delta T)$. Thermal conductivity is a material property that is primarily dependent on the medium's phase, temperature, density, and molecular bonding. The thermal conductivity of a material generally varies with temperature but the variation is usually small over a wide range of temperatures. Table I shows the conductivity values for some substances.

Fourier's law states that the time rate of heat transfer or heat transmitted per unit area and per unit time, represented as the vector $\mathbf{q}$, through a material is proportional to the negative gradient of temperature $\nabla T$,

$$
\mathbf{q}=-k \nabla T
$$

where $k$ is the thermal conductivity of the material. For many simple applications, Fourier's law is used in its one-dimensional form 


$$
I_{x}=-k S \frac{d T}{d x}
$$

where $I_{x}$ is the rate of heat flow in the $x$-direction $\left(I_{x}=d Q / d t\right)$ and $S$ is the surface area of the cross-section.

\subsection{Steady state conduction}

Steady state conduction occurs when the temperature difference driving the conduction is constant so that after an equilibration time, the spatial distribution of temperatures in the conducting object does not change any further. For example, a bar may be cold at one end and hot at the other, but the temperature gradient along the bar does not change with time. The temperature at any given section of the rod remains constant, and this temperature varies linearly along the direction of heat transfer [3]. In steady state conduction, the amount of heat entering a section is equal to the amount of heat coming out. In steady state conduction, all the laws of direct current electrical conduction can be applied to "heat currents". In such cases, it is possible to take "thermal resistances" as analogues of electrical resistances. Temperature plays the role of voltage and heat transferred is the analogue of electrical current.

Integrating equation (2) for a homogeneous material of one-dimensional geometry between two endpoints at constant temperature gives the following expression for the heat flow ratio

$$
I_{x}=\frac{\Delta Q}{\Delta t}=-k S \frac{\Delta T}{\Delta x}=-k S \frac{T_{2}-T_{1}}{L}
$$

where $\Delta T$ is the temperature difference between the ends and $\Delta x$ is the distance between the ends. This law forms the basis for deriving the heat equation. Ohm's law is the electrical analogue of Fourier's law

$$
\Delta T=-R I, \quad T_{1}-T_{2}=R I
$$

where $R$ is the thermal resistance defined as follows 


$$
R=\frac{\Delta x}{k S}
$$

\subsection{Conduction through hollow cylinders}

Figure 1 represents a section of a cylindrical tube made of a material whose thermal conductivity is $k$ and length $L$, where $r_{1}$ and $r_{2}$ are the inner and outer radii of the hollow circular section. The temperature difference between the inner and outer wall can be expressed as $T_{2}-T_{1}$, and the area of the heat flow is $S_{r}=2 \pi r L$. In the steady state regime, the heat flux through the surface of a cylinder layer of radius $r$ is

$$
I_{r}=-k S_{r} \frac{d T}{d r}=-2 \pi k r L \frac{d T}{d r} \quad \text { and } \quad I_{r} \frac{d r}{r}=-2 \pi k L d T
$$

and integrating between the limits $r_{1}$ and $r_{2}$ we obtain

$$
I_{r} \int_{r_{1}}^{r_{2}} \frac{d r}{r}=-2 \pi k L \int_{T_{1}}^{T_{2}} d T
$$

Therefore the rate of heat transfer is

$$
I_{r}=2 \pi k L \frac{T_{1}-T_{2}}{\ln \left(r_{2} / r_{1}\right)}
$$

and the thermal resistance for the hollow cylinder is therefore

$$
R=\frac{\ln \left(r_{2} / r_{1}\right)}{2 \pi k L}
$$

and the heat flux can be written as follows 


$$
I_{r}=2 \pi k L r_{m} \frac{T_{1}-T_{2}}{r_{2}-r_{1}}
$$

where $r_{m}$ is the log-mean radius

$$
r_{m}=\frac{r_{2}-r_{1}}{\ln \left(r_{2} / r_{1}\right)}
$$

\section{Experimental apparatus description}

In this laboratory practice, we used several hand-made hollow cylinders (Figure 2) of different materials such as wood, plastic, steel and aluminium, in order to check the thermal conductivity using Fourier's law under the steady state conduction regime. To do this, an experimental apparatus was designed and built in our laboratory in order to carry out the practical application of heat conduction through hollow cylinders and determine the thermal conductivities of the different materials considered. A photograph of the experimental apparatus is shown in Figure 3. Our hand-made thermal device includes a linear electric resistance in an aluminium case, as can be seen in Figure 4 and it is possible to regulate the heat flux by means of the electronic regulator shown in Figure 5. We can calculate how much heat is dissipated by the resistance by measuring the current intensity and voltage.

The hollow cylinder of the material is placed in the aluminium case of the resistance and at time cero the electric switch is turned on and the resistance starts heating. Both the temperature on the external surface of the aluminium case and that on the surface of the cylinder are measured using a conventional thermometer with a copper cap to make a better contact (Figure 6). The temperature values are monitored until the steady state regime is reached when the temperatures are approximately constant. When the temperature values are near those of the steady state regime we use equation (8) in order to obtain the value of thermal conductivity.

\section{Results and discussion}


Figure 7 shows the plot of temperature evolution as a function of time for a wooden cylinder and for the following values of intensity and voltage, $i_{e f}=0.48 \pm 0.01 \mathrm{~A}, V_{e f}=$ $37.0 \pm 0.1 \mathrm{~V}$, respectively. Heating is stopped at $t=33$ minutes because the wood starts to decompose due to the high temperatures reached at the surface of the aluminium case. For this material $T_{1}-T_{2}=35 \pm 2{ }^{\circ} \mathrm{C}$ in the steady state. The thermal conductivity obtained with the help of equation (8) is $k=0.13 \pm 0.01 \mathrm{~W} \mathrm{~m}^{-1} \mathrm{~K}^{-1}$. Table I shows the values given in the bibliography. For wood (in general) the value for $k$ is $0.13 \mathrm{~W} \mathrm{~m}^{-1} \mathrm{~K}^{-1}$, while for pinewood, $k$ $=0.16 \mathrm{~W} \mathrm{~m}^{-1} \mathrm{~K}^{-1}$. With this experiment it would be possible to distinguish between different types of wood when there is a sufficient difference in their thermal conductivity values. The difference in the $\mathrm{k}$ values for resinous and non-resinous woods could be sufficient to be distinguished by means of this method.

In Figure 8 we plot the temperature evolution as a function of time for a plastic cylinder (polyethylene) for $i_{e f}=0.50 \pm 0.01 \mathrm{~A}$ and $V_{e f}=44.3 \pm 0.1 \mathrm{~V}$. The $i_{e f}$ and $V_{e f}$ values (used to determine the thermal flux) are selected in order to get a relatively quick heating time, avoiding reaching the melting point of the material. In this experiment heating is stopped when the steady state regime is reached but it is necessary to take into account that a high temperature at the external surface of the resistance case could melt the material of the cylinder. It is advisable to conduct the experiment slowly (low $i_{e f}$ and $V_{e f}$ values) in order to obtain a low temperature in the steady state regime. For this material $T_{1}-T_{2}=34 \pm 2{ }^{\circ} \mathrm{C}$ in the steady state. The thermal conductivity obtained is $k=0.33 \pm 0.01 \mathrm{~W} \mathrm{~m}^{-1} \mathrm{~K}^{-1}$, whereas the thermal conductivity of polyethylene (LDPE) is $k=0.300-0.335 \mathrm{~W} \mathrm{~m}^{-1} \mathrm{~K}^{-1}$ (see Table I). Again, this experiment could be used to differentiate between different materials with a similar external appearance. For example, teflon ${ }^{\circledR}$ and polyethylene HD are both white in colour and have a similar external appearance but very different $k$ values.

Figure 9 shows the temperature evolution as a function of time for two metallic cylinders: aluminium $\left(i_{e f}=0.70 \pm 0.01 \mathrm{~A}, V_{e f}=64.0 \pm 0.1 \mathrm{~V}\right)$ and steel $\left(i_{e f}=0.54 \pm 0.01 \mathrm{~A}, V_{e f}=\right.$ $48.3 \pm 0.1 \mathrm{~V})$. It may be seen that the $T_{1}$ and $T_{2}$ temperature values for the two cylinders are very similar due to the high $k$ values for aluminium and steel, but the values of $k$ obtained using equation (8) are lower than 1 , which are very different from the actual values for these materials (see Table I [10-12]). The high $k$ values for steel and aluminium imply a high 
temperature at the outer wall of the cylinders; therefore, heat transfer by convection may be important and it is not possible to measure it in this manner.

We use an external hollow cylinder of wood in order to minimize convection and obtain a better value of $k$ for aluminium. In these conditions we have a heat transfer with thermal resistances in series, with a constant heat transfer through both materials: inner aluminium cylinder and outer wooden cylinder $\left(I=I_{A l}=I_{\text {wood }}\right)$. Fourier's law is expressed now as

$$
T_{1}-T_{2}=R_{e q} I
$$

and

$$
R_{e q}=R_{A l}+R_{\text {wood }}
$$

where each thermal resistance is calculated using equation (9). Figure 10 shows the temperature evolution versus time for the two thermal resistances in series, internal aluminium and external wood cylinders. Now, the $T_{1}$ and $T_{2}$ temperature values are closer in the steady state due to the small contribution of convection. Therefore, the value obtained for thermal conductivity $k_{\mathrm{Al}}=1.92 \pm 0.01 \mathrm{~W} \mathrm{~m}^{-1} \mathrm{~K}^{-1}$ is higher than that without the external wood cylinder but lower than the value in the bibliography (Table I). The explanation for this result is as follows. In the steady state the temperature difference is $T_{1}-T_{2}=1 \pm 2{ }^{\circ} \mathrm{C}$. This value matches the precision of the thermometer $\left(1^{\circ} \mathrm{C}\right)$ but we need a precision of $0.01^{\circ} \mathrm{C}$ in order to obtain a $k$ value close to that found in the bibliography due to the small difference between temperature values.

\section{Conclusions}

A simple experimental apparatus was designed and built, which can be used in practical lectures on heat conduction for students at the intermediate university level. The experimental procedure shown in this paper makes it possible to obtain the thermal conductivity for different materials, which are considered thermal insulators: plastics and wood. In the case of materials with high thermal conductivity such as metals, it is necessary to minimize convection at the outer surface of the material and use high precision thermometers. The problem of heat conduction in a hollow cylinder considered in this paper 
illustrates a number of concepts, which help students to understand this topic better. They can easily verify how the system evolves toward a steady state, characterized by a constant temperature difference between the inner and outer walls of the hollow cylinder. When the system reaches this steady state, students can easily determine the thermal conductivity of the material of which the cylinder is made. Students can also verify that the values for the conductivities they obtain for thermal insulators (wood and plastic) agree with those found in the bibliography. However, in the case of metals the conductivity values they obtain do not agree with the correct values and they can understand the reasons for this disagreement

\section{Acknowledgements}

This work was supported by the 'Vicerrectorado de Tecnología e Innovación Educativa' of the University of Alicante, Spain (GITE-09006-UA) and by the Generalitat Valenciana of Spain under project PROMETEO/2011/021.

\section{References}

[1] Brun J L and Pacheco A F 2005 Reducing the heat transfer through a wall Eur. J. Phys. $2611-8$

[2] González M I and Lucio J H 2008 Investigating convective heat transfer with an iron and a hairdryer Eur. J. Phys. 29 263-73

[3] Incropera F P and De Witt D P 1990 Fundamentals of Heat and Mass Transfer $3^{\text {rd }}$ edn (New York: Wiley)

[4] Díaz-Aguilera A 1990 On heat conduction in one-dimensional solids Am. J. Phys. 58 $779-80$

[5] Ràfois I and Ortín J 1992 Heat conduction in a metallic rod with Newtonian losses $A m$. J. Phys. 60 846-52.

[6] Bee G, Ballentine K and Thomsen M 2008 Realistic problems involveg thermal conductivity Am. J. Phys. 76 970-4 
[7] Brody J, Andreae P and Robinson C A 2010 A simple optical probbe of transient heat conduction Am. J. Phys. 78 529-31

[8] Laufer G, Rotchford N B and Krauss R H 1997 Temperature visualization in conducting solids using thermographic phosphors Am. J. Phys. 65 447-9

[9] Bacon M E, Wick R M and Hecking P 1995 Heat, light and videotapes: Experiments in heat conduction using liquid crystals Am. J. Phys. 63 359-63

[10] http://www.engineeringtoolbox.com/thermal-conductivity-d_429.html

[11] http://www.matbase.com/

[12] www.miliarium.com 


\section{FIGURE CAPTIONS}

Figure 1.- Heat conduction through a hollow cylinder: Cross section of the cylinder and direction of the heat flux.

Figure 2.- Hand-made hollow cylinders of different materials: wood, plastic, aluminium and iron.

Figure 3.- Experimental set-up.

Figure 4.- Hand-made thermal device which is introduced inside the hollow cylinder.

Figure 5.- Electronic regulator, ammeter and voltmeter.

Figure 6.- Thermometer in the cylinder and detail of the copper cap.

Figure 7.- Temperature evolution versus time for a wooden cylinder $\left(r_{1}=25.30 \pm 0.01 \mathrm{~mm}\right.$, $\left.r_{2}=50.00 \pm 0.01 \mathrm{~mm}, L=230 \pm 1 \mathrm{~mm}, i_{e f}=0.48 \pm 0.01 \mathrm{~A}, V_{e f}=37.0 \pm 0.1 \mathrm{~V}\right) . T_{1}$ is the temperature at the surface of the aluminium case and $T_{2}$ is the temperature at the outer wall of the wooden cylinder. $T_{1}-T_{2}=35 \pm 2{ }^{\circ} \mathrm{C}$ in the steady state.

Figure 8.- Temperature evolution versus time for a polyethylene cylinder $\left(r_{1}=25.30 \pm 0.01\right.$ $\left.\mathrm{mm}, r_{2}=46.10 \pm 0.01 \mathrm{~mm}, L=230 \pm 1 \mathrm{~mm}, i_{e f}=0.50 \pm 0.01 \mathrm{~A}, V_{e f}=44.3 \pm 0.1 \mathrm{~V}\right)$. $T_{1}$ is the temperature at the surface of the aluminium case and $T_{2}$ is the temperature at the outer wall of the cylinder of polyethylene. $T_{1}-T_{2}=34 \pm 2{ }^{\circ} \mathrm{C}$ in the steady state.

Figure 9.- Temperature evolution versus time for metallic cylinders: aluminium $\left(r_{1}=\right.$ $25.40 \pm 0.01 \mathrm{~mm}, r_{2}=35.00 \pm 0.01 \mathrm{~mm}, L=230 \pm 1 \mathrm{~mm} i_{e f}=0.70 \pm 0.01 \mathrm{~A}, V_{e f}=$ $64.0 \pm 0.1 \mathrm{~V})$ and steel $\left(r_{1}=25.30 \pm 0.01 \mathrm{~mm}, r_{2}=36.20 \pm 0.01 \mathrm{~mm}, L=230 \pm 1 \mathrm{~mm}\right.$ $\left.i_{e f}=0.54 \pm 0.01 \mathrm{~A}, V_{e f}=48.3 \pm 0.1 \mathrm{~V}\right) . T_{1}$ is the temperature at the surface of the aluminium case and $T_{2}$ is the temperature at the outer wall. For the aluminium 
cylinder, $T_{1}-T_{2}=19 \pm 2{ }^{\circ} \mathrm{C}$ in the steady state and for steel cylinder, $T_{1}-T_{2}=$ $8 \pm 2{ }^{\circ} \mathrm{C}$ in the steady state.

Figure 10.- Temperature evolution versus time for the aluminium cylinder for two thermal resistances in series, aluminium $\left(r_{1}=25.40 \pm 0.01 \mathrm{~mm}, r_{2}=35.0 \pm 0.01 \mathrm{~mm}, L=\right.$ $230 \pm 1 \mathrm{~mm})$ and external wooden $\left(r_{1}=35.30 \pm 0.01 \mathrm{~mm}, r_{2}=51.0 \pm 0.01 \mathrm{~mm}, L=\right.$ $230 \pm 1 \mathrm{~mm}$ ) cylinders. $T_{1}$ is the temperature at the surface of the case and $T_{2}$ is the temperature at the outer wall of the aluminium cylinder. $T_{1}-T_{2}=1 \pm 2{ }^{\circ} \mathrm{C}$ in the steady state. 


\section{FIGURE 1}

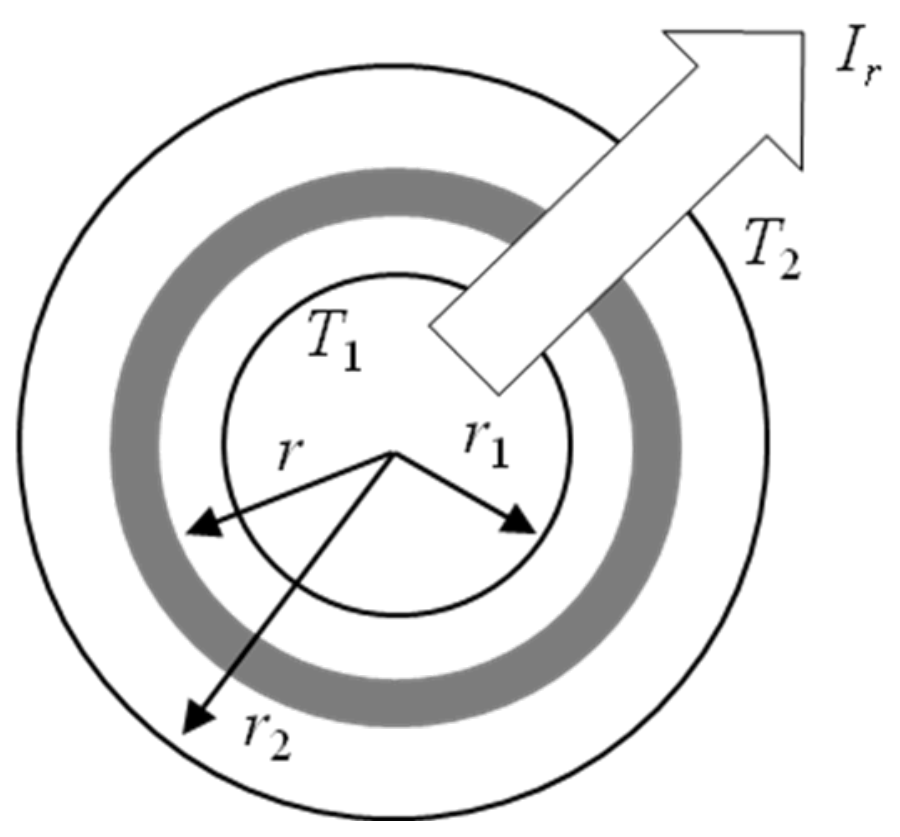


M. Ortuño, A. Márquez, S. Gallego, C. Neipp and A. Beléndez, "An experiment in heat conduction using hollow cylinders", European Journal of Physics, Vol. 32, No 4, 1065-1075 (2011).

\section{FIGURE 2}

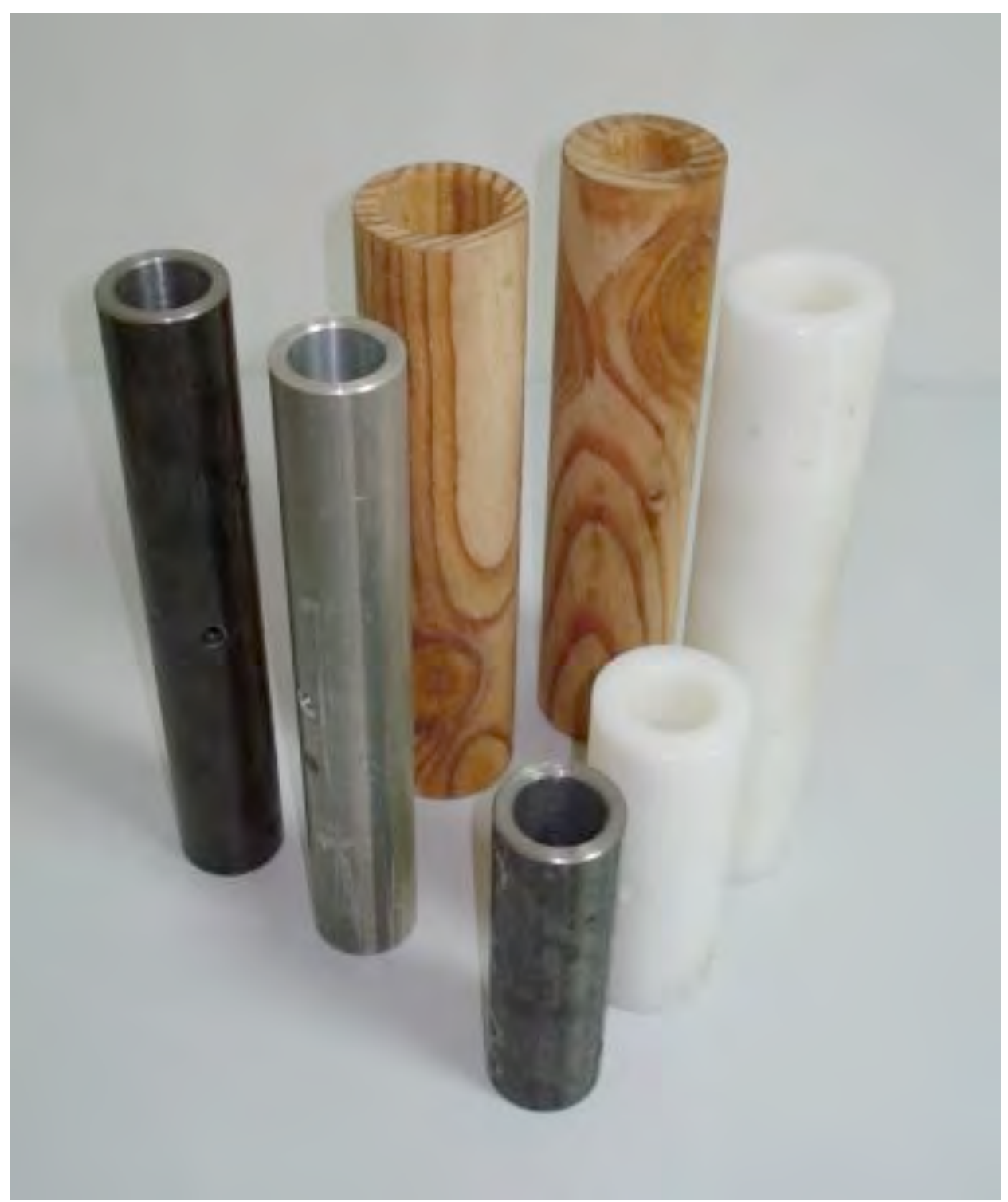


M. Ortuño, A. Márquez, S. Gallego, C. Neipp and A. Beléndez, "An experiment in heat conduction using hollow cylinders", European Journal of Physics, Vol. 32, No 4, 1065-1075 (2011).

\section{FIGURE 3}

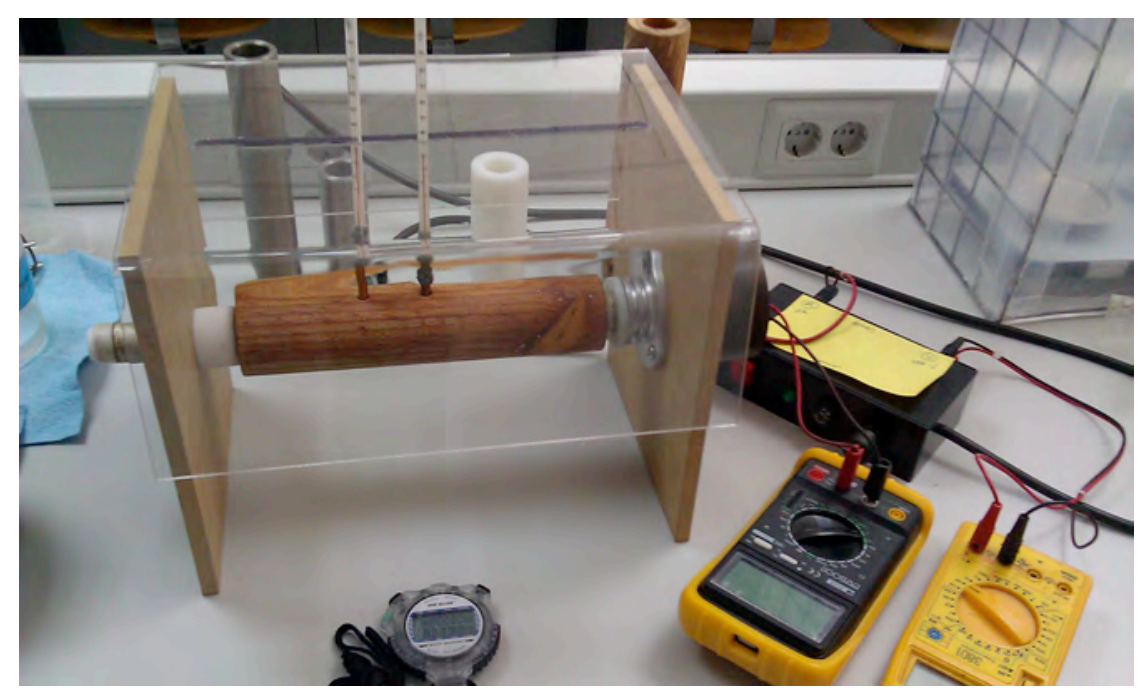


M. Ortuño, A. Márquez, S. Gallego, C. Neipp and A. Beléndez, "An experiment in heat conduction using hollow cylinders", European Journal of Physics, Vol. 32, No 4, 1065-1075 (2011).

\section{FIGURE 4}

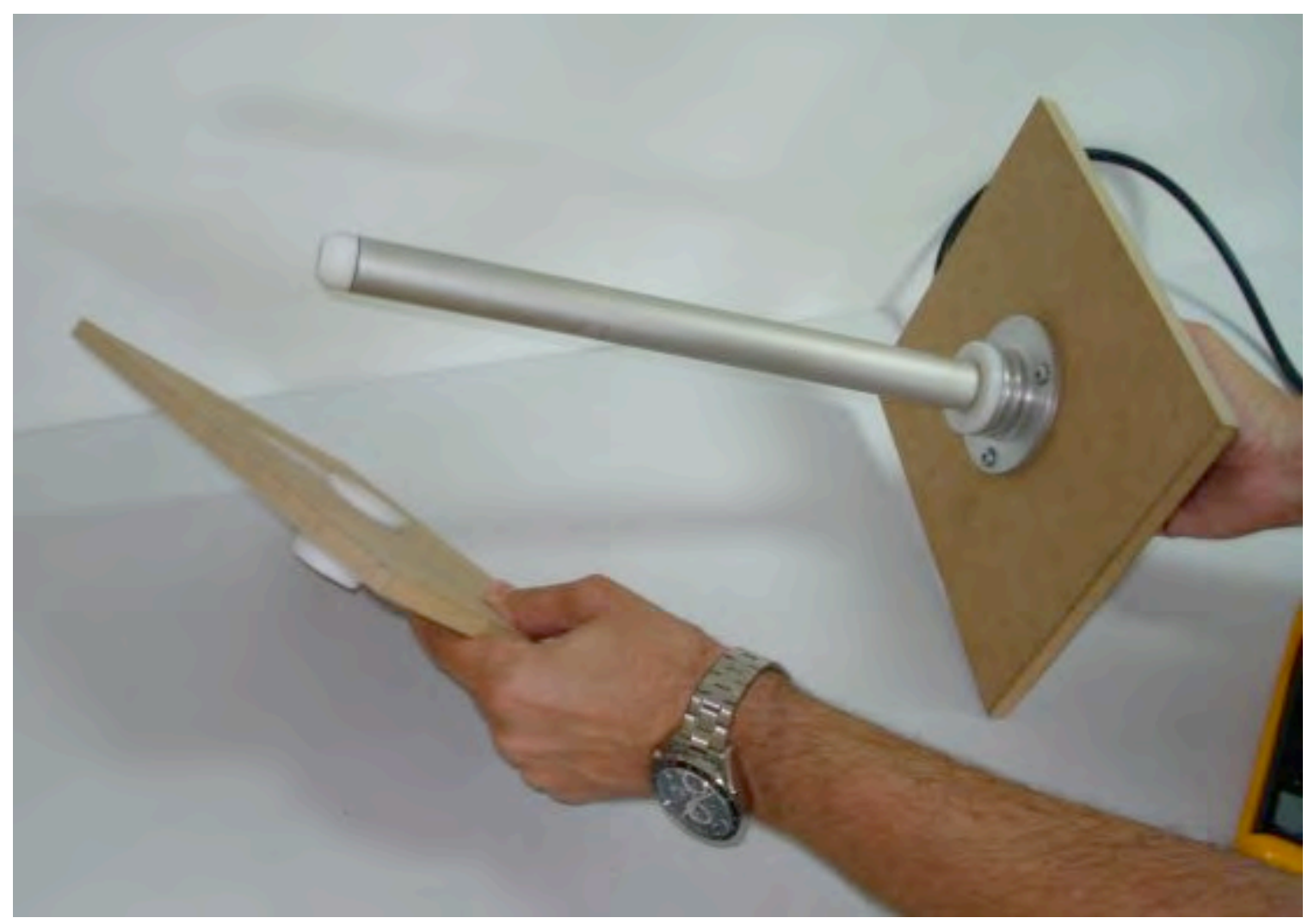


M. Ortuño, A. Márquez, S. Gallego, C. Neipp and A. Beléndez, "An experiment in heat conduction using hollow cylinders", European Journal of Physics, Vol. 32, No 4, 1065-1075 (2011).

\section{FIGURE 5}

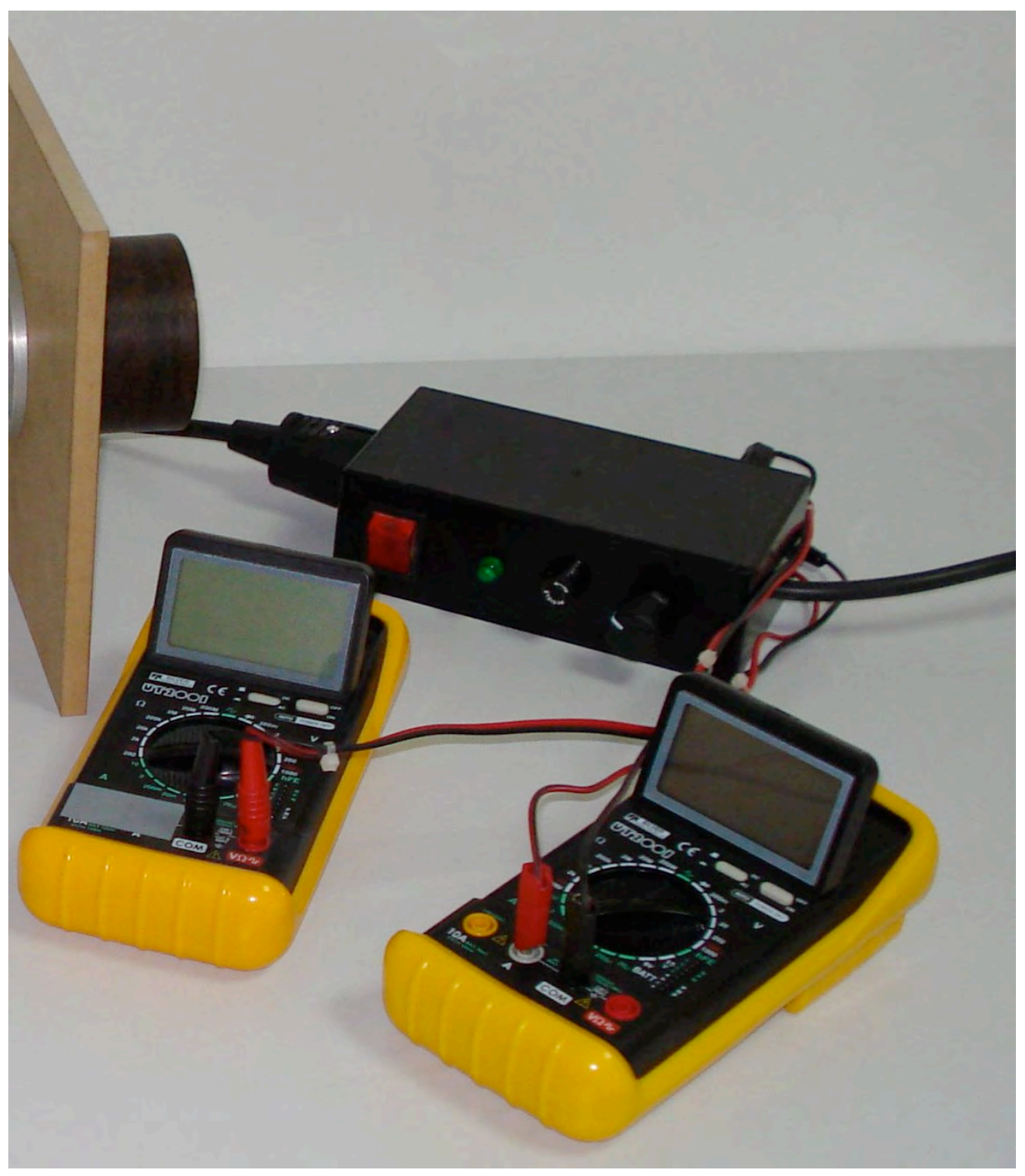


M. Ortuño, A. Márquez, S. Gallego, C. Neipp and A. Beléndez, "An experiment in heat conduction using hollow cylinders", European Journal of Physics, Vol. 32, No 4, 1065-1075 (2011).

\section{FIGURE 6}
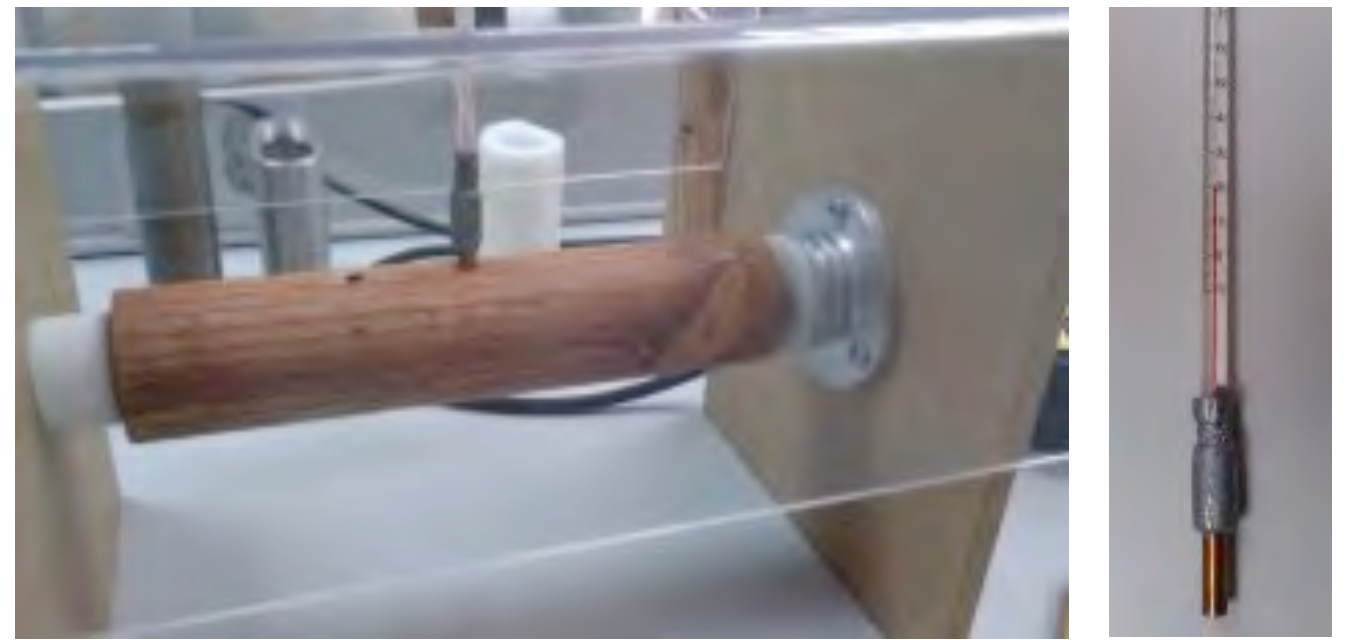


\section{FIGURE 7}

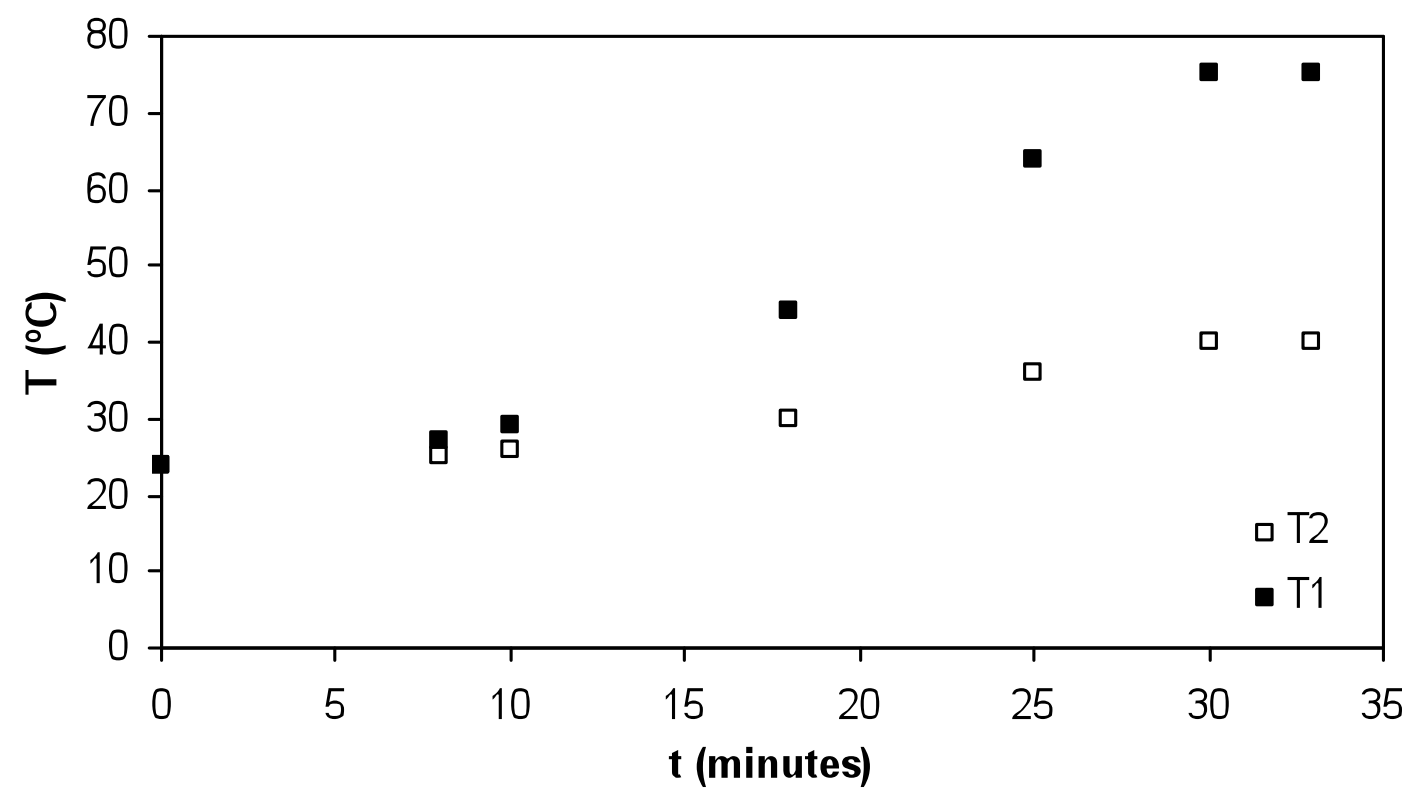




\section{FIGURE 8}

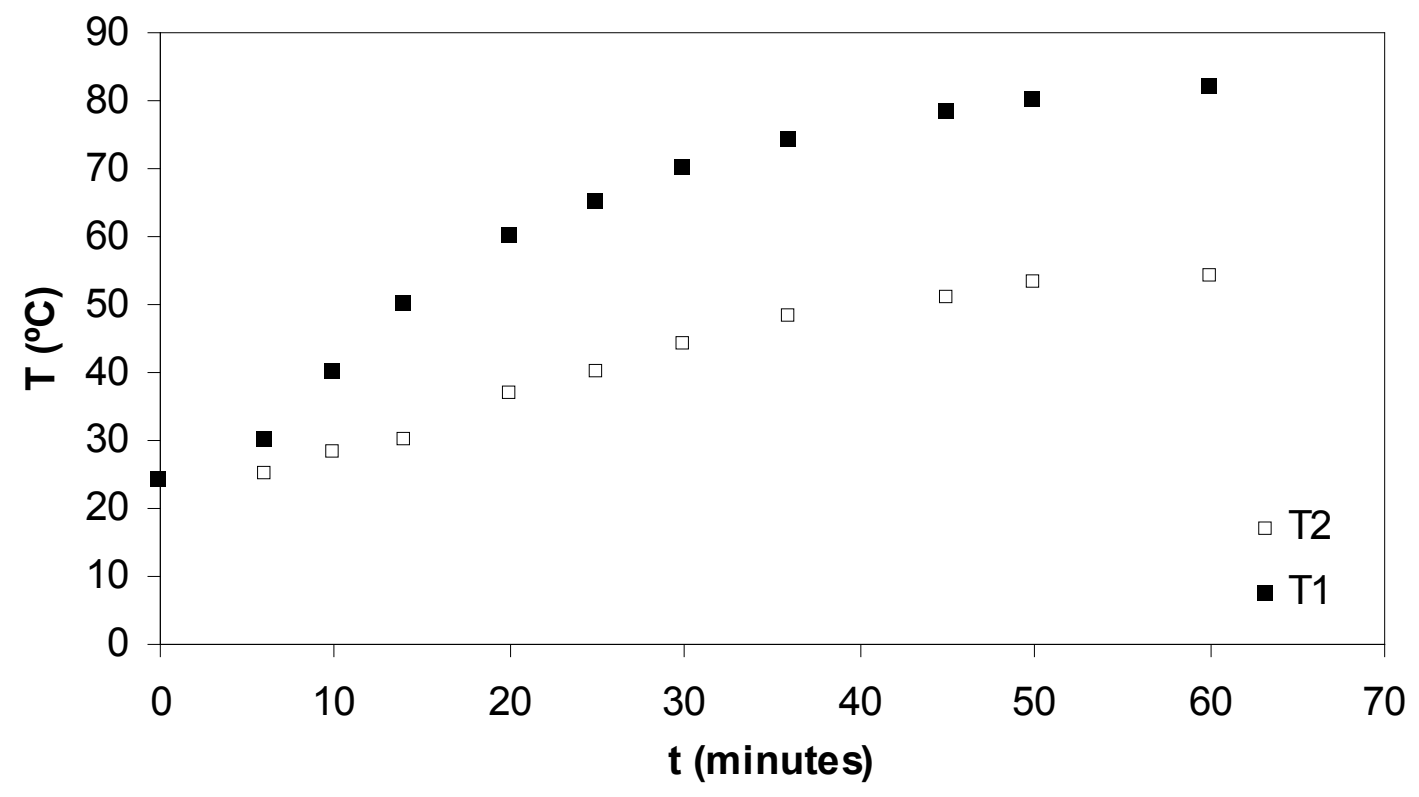




\section{FIGURE 9}

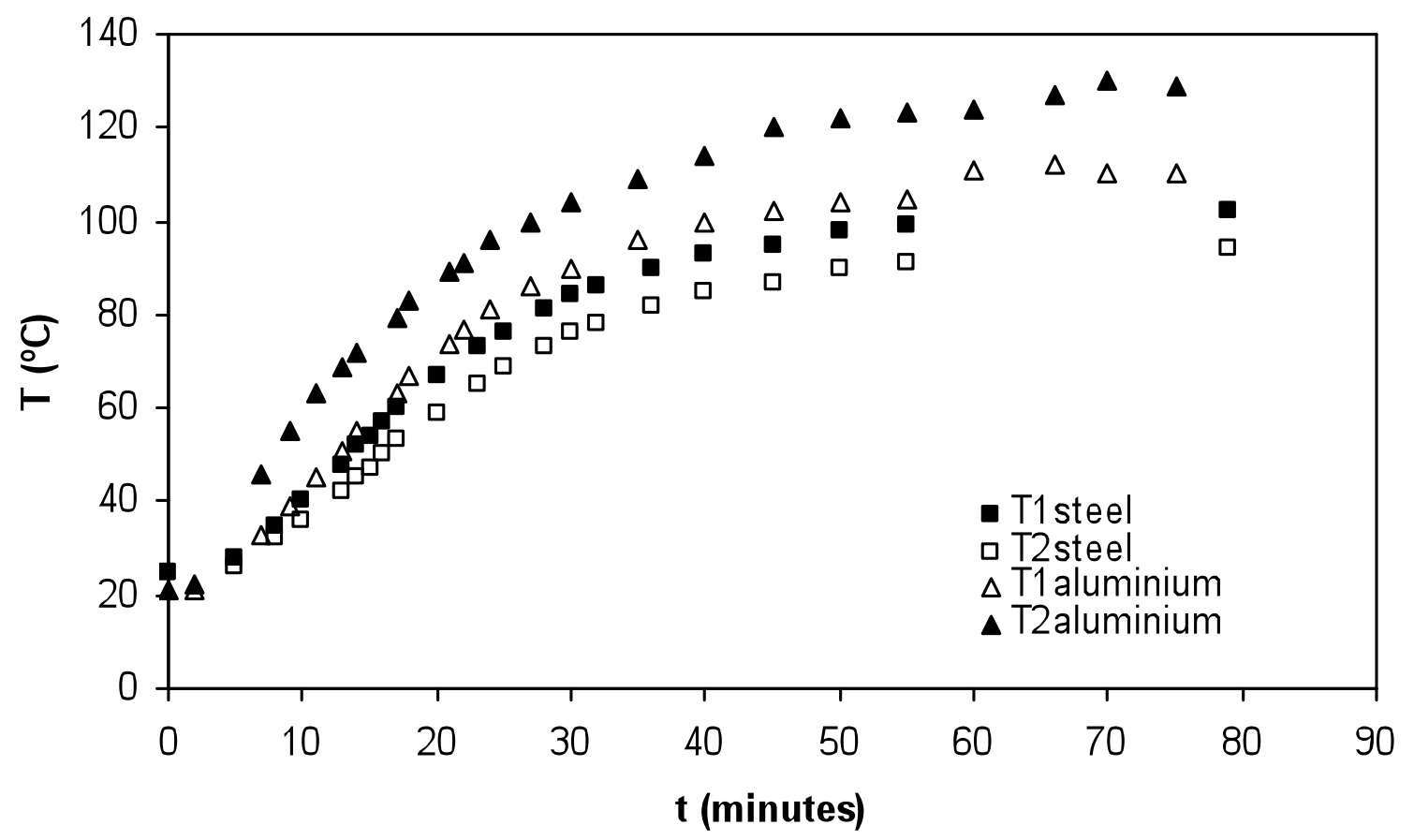


FIGURE 10

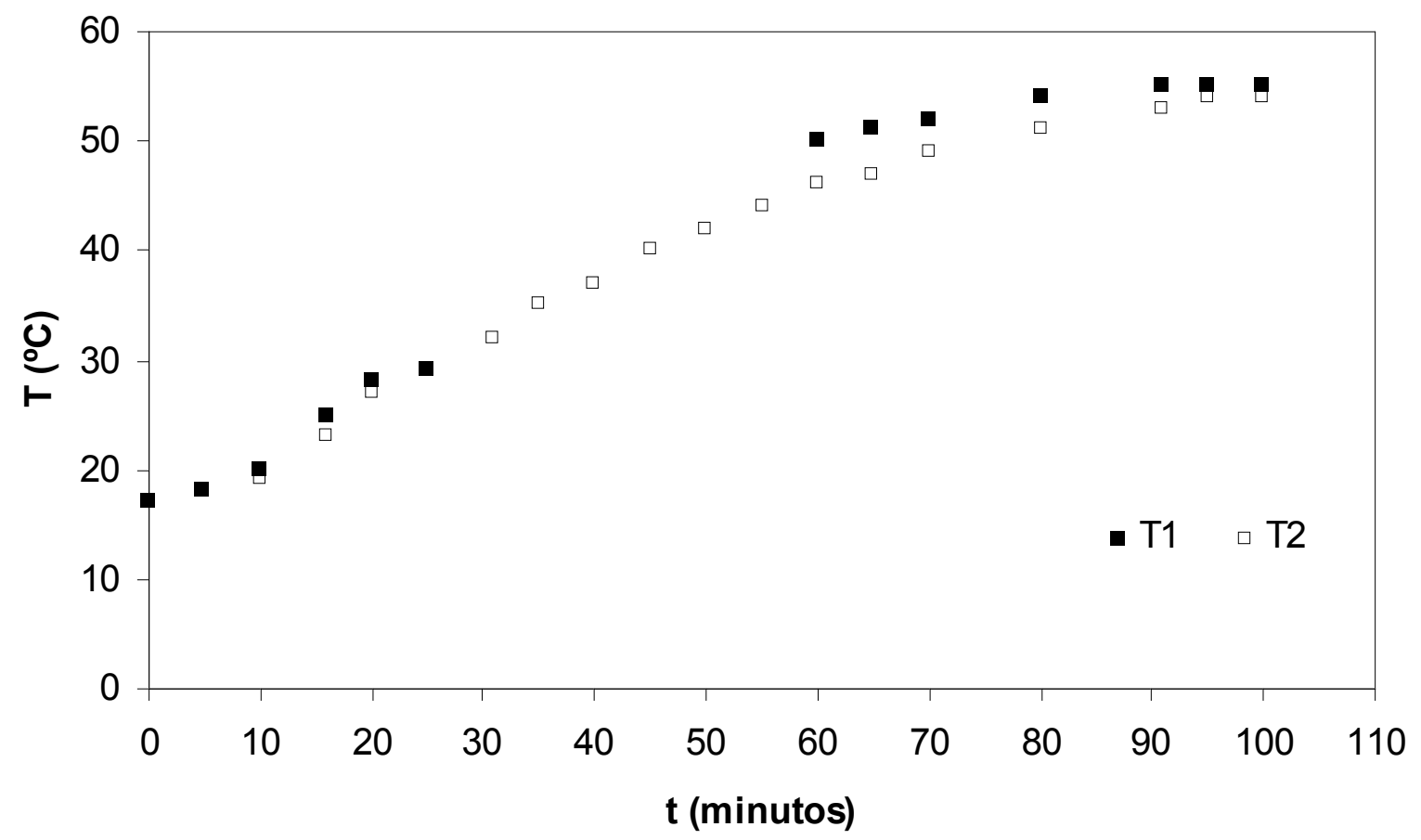




\section{TABLE I}

Thermal conductivity coefficients for several materials [10-12]

\begin{tabular}{|l|c|}
\hline \multicolumn{1}{|c|}{ Material } & $k\left(\mathrm{~W} \mathrm{~K}^{-1} \mathrm{~m}^{-1}\right)$ \\
\hline Aluminium & 250 \\
\hline Steel & $47-58$ \\
\hline Polyethylene (HD) & $0.42-0.51$ \\
\hline Polyethylene (LDPE) & $0.300-0.335$ \\
\hline Teflon $®$ & 0.23 \\
\hline Maple wood & 0.35 \\
\hline Pine wood & 0.16 \\
\hline Air & 0.025 \\
\hline
\end{tabular}

